PHYSICAL REVIEW B 85, 035301 (2012)

\title{
Light-trapping plasmonic nanovoid arrays
}

\author{
Ricky B. Dunbar, ${ }^{1}$ Holger C. Hesse, ${ }^{1}$ Dominik S. Lembke, ${ }^{1}$ and Lukas Schmidt-Mende ${ }^{1,2, *}$ \\ ${ }^{1}$ Department of Physics and Center for NanoScience (CeNS), Ludwig-Maximilians University, Amalienstrasse 54, 80799 Munich, Germany \\ ${ }^{2}$ Department of Physics, University of Konstanz, Universitätsstrasse 10, 78457 Constance, Germany \\ (Received 22 June 2011; revised manuscript received 29 November 2011; published 3 January 2012)
}

\begin{abstract}
We consider the suitability of metallic nanovoid arrays for confining incident light and enhancing absorption in an adjacent absorbing material, such as an organic semiconductor. Such nanostructures can facilitate strong coupling of incident light into plasmonic modes localized at the surface of the metal. We investigate this system both experimentally and by performing numerical calculations. To fabricate the samples, we employ a novel imprinting technique to obtain large-area, highly ordered metallic nanovoid arrays. Strong overall absorption enhancements are measured for nanostructured samples compared to planar samples for interfaces between silver and a common organic semiconductor, phenyl- $\mathrm{C}_{61}$-butyric acid methyl ester. Full-wave, three-dimensional finite element method simulations are employed to investigate the nature of this light trapping, and very good agreement is observed between experimental and simulated absorption spectra. The simulations reveal highly localized surface modes and indicate that the majority of the additional absorption occurs within the organic semiconductor.
\end{abstract}

DOI: 10.1103/PhysRevB.85.035301

PACS number(s): 81.07.Pr, 81.05.Fb, 73.20.Mf

\section{INTRODUCTION}

The propagation of light can be dramatically altered upon interaction with a metallic nanostructure. The nature of this alteration is largely governed by the geometry of the metal nanostructure; therefore, control over the geometry translates directly into control over light propagation. In recent years, the motivation of obtaining control over light propagation on the nanometer scale has stimulated a vast amount of exciting research in the field of optoelectronics. Light transmitted through a thin metallic film featuring an array of subwavelength apertures can exceed the amount of light incident on the total aperture area. ${ }^{1}$ This effect is widely reported to be enabled by tunneling of grating-coupled surface plasmon polaritons (SPPs, surface-bound electromagnetic waves which can propagate along metal-dielectric interfaces). Raman scattering from single molecules and nanoparticles in the direct environment of nanostructured silver surfaces can be detected with enhancement factors of up to the order of $10^{14}$, which is attributed to plasmonic field enhancement near the silver interface. ${ }^{2}$ In solar cells, absorption can be enhanced by increasing the optical path length in the active layer. This can be achieved, for example, by exciting scattering or waveguide modes or by trapping it via coupling to SPPs or localized surface plasmons ${ }^{3,4}$ (LSPs, nonpropagating excitations of conduction electrons within a metallic nanostructure). Metallic nanoparticles deposited on the front surface of solar cells have been shown to enhance power conversion efficiency through favorable forward scattering into the absorbing medium., Efficiency enhancements have also been reported for devices which feature nanoparticle layers adjacent to the absorbing medium (separated by or contained in a thin spacing layer) on the front side. The enhancements were attributed to enhanced absorption in the volume of semiconductor located within close (within the nominal volume over which the near-field energy density enhancement associated with LSPs can be considered to extend) proximity to the particles. ${ }^{7,8}$ Back electrodes featuring arrays of nanowires or nanovoids are excellent candidates for obtaining control over light propagation in optoelectronic devices via grating-coupled SPP excitation, scattering, LSP excitation or a combination of these processes. ${ }^{3,4}$ The dominance of each mode is governed by the geometry. Flat metallic surfaces can host SPP modes excited from normal incidence providing appropriate scattering structures (typically grooves or ridges) are present for in-coupling of incident light. If these structures are metallic and of the order of $20 \mathrm{~nm}$ or more in height, significant excitation of LSP modes can occur as well. ${ }^{9}$ Angle- and orientationdependent reflectometry has been successfully implemented to distinguish between SPP, LSP, and hybrid modes, which can be readily excited on metallic nanovoid arrays. ${ }^{10}$

In this study, high-order nanovoid arrays are investigated for light-trapping applications in organic solar cells. Organic solar cells, as well as other thin-film photovoltaic technologies based on materials such as amorphous silicon and GaAs, stand to benefit greatly from plasmonic light trapping, as it would enable the construction of devices with thinner active layers (with reduced series resistance) without compromising total light absorption. This would correspond to a simultaneous increase in power conversion efficiency and a reduced consumption of the active material.

In order to fabricate highly ordered plasmonic structures across large areas with precise control over the geometry, we employ an imprinting technique. Here, an anodized aluminum oxide (AAO) stamp featuring highly ordered hexagonal pore geometry is used to imprint an organic film, forming an array of freestanding organic nanowires. A thick layer of metal (more than $200 \mathrm{~nm}$, considerably larger than the typical height of the nanowires) is deposited on top via thermal evaporation. As the deposited silver structurally conforms to the underlying organic nanowire array, a metal-organic nanovoid interface is obtained. With this technique, the ordering, periodicity, and diameter of the organic nanowires and hence the metallic nanovoids can be determined by adjusting the geometry of the AAO pore array. The pore diameter can be conveniently determined using a pore-widening step, and the pore periodicity can be set to a value within a broad range of $50-420 \mathrm{~nm} .{ }^{11}$ 
The height of the organic nanowires, corresponding to the depth of the nanovoids, is largely determined by the imprinting pressure, temperature, and duration. The flexibility of this technique allows the fabrication of a wide variety of nanovoid array geometries, which can be adjusted to be application specific. In addition, this technique requires no electronbeam lithography, which is an expensive high-end technology with limited application for large-area manufacturing. The stamps used in this study are produced by anodization, which is a simple, fully scalable, and inexpensive technique that can be immediately incorporated into commercial solar cell fabrication. In fact, it is conceivable that this technique could be incorporated into roll-to-roll assembly. Previous reports on the fabrication of structured organic interfaces via imprinting, which include a number in which AAO stamps were used, ${ }^{12,13}$ demonstrate the potential of this technique. ${ }^{14,15}$ These studies were primarily focused on using imprinting to improve the morphology of the interface in bulk heterojunction solar cells.

\section{SAMPLE PREPARATION}

Two stamps are fabricated with pore-pore periodicities of 60 (stamp A) and $100 \mathrm{~nm}(\operatorname{stamp} B)$. Mechanically polished Al foils (99.999\%, Goodfellow) first undergo electrochemical polishing in a 1:3 volume ratio solution of perchloric acid in ethanol (>95\%) for 8 minutes under a bias of $20 \mathrm{~V}$. The polished foils are then anodized in a two-step anodization process. ${ }^{11,16}$ The pore periodicity can be controlled by varying the electrolyte and the potential under which the anodization is carried out. Stamp $A$ is produced by anodizing polished $\mathrm{Al}$ foil in sulfuric acid $(0.3 \mathrm{M})$ at a potential of $25 \mathrm{~V}$. The anodization for stamp $B$ is carried out in oxalic acid $(0.3 \mathrm{M})$ at a potential of 40 $\mathrm{V}$. The porous oxide is then removed in a solution of phosphoric (6 wt.\%) and chromic (1.8 wt.\%) acid, leaving a highly ordered aluminum surface. The first anodization and oxide removal steps have the function of patterning the aluminum foil in preparation for the second anodization step, which is conducted for 1 and 5 minutes for stamps $A$ and $B$, respectively. The resulting pores in the aluminum oxide membranes are then widened in phosphoric acid (5 wt.\%) for 15 (stamp A) and 30 minutes (stamp $B$ ). The resulting membranes are then placed in a desiccator where a small droplet of $\mathrm{F}_{13}$-TCS silane solution placed by the stamps is allowed to evaporate. ${ }^{17} \mathrm{~A}$ thin silane layer is formed on the surface of the stamps, which acts to reduce the adhesion with the organic material during imprinting. A scanning electron microscopy (SEM) image of stamp $A$ is shown in Fig. 1(a), and a side-on schematic is shown in Fig. 1(e). Phenyl- $\mathrm{C}_{61}$-butyric acid methyl ester (PCBM) films are spin coated on $2 \mathrm{~cm}^{2}$ glass substrates. The silanized AAO membrane is cleaned via ultrasonification in chlorobenzene for 5 minutes, rinsed in isopropanol, and dried with a stream of nitrogen. It is then used to imprint the PCBM film in a mechanical hot press at a pressure of 250 bar for 5 minutes at $120^{\circ} \mathrm{C}$. All samples are fabricated in duplicate: once on quartz glass substrates and once on conducting indium tin oxide- (ITO) covered glass substrates. The samples on ITO are used for SEM imaging whilst the ITO-free layers are used for spectroscopy. A thin layer of ITO is undesirable in a

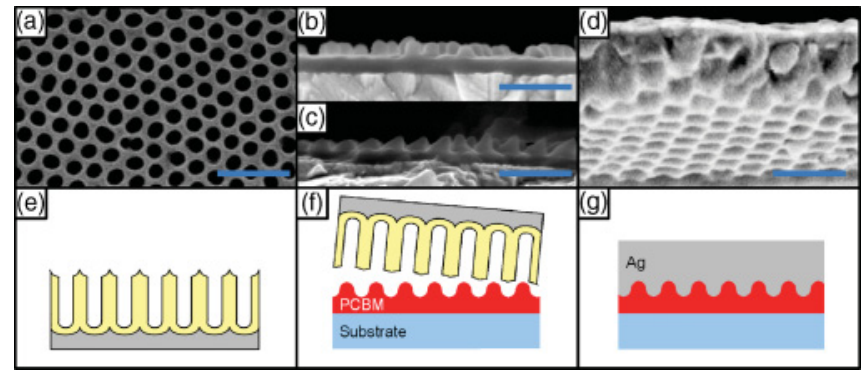

FIG. 1. (Color online) Sample preparation (a)-(d) SEM images. All scale bars are $200 \mathrm{~nm}$ long. (a) Top view of a stamp: highly ordered porous AAO. The hexagonal packing is evident. (b) Sample A, PCBM nanowire array produced after imprinting a PCBM film with a stamp with a 60-nm pore periodicity. (c) Sample $B$, PCBM nanowire array produced after imprinting a PCBM film with a stamp with a $100-\mathrm{nm}$ pore periodicity. (d) Ordered silver nanovoid array, as observed from underneath (the PCBM nanowires have been removed in this position, see text). (e)-(g) Schematic of sample preparation. (e) Side-on view of a finished stamp. (f) Imprinting of a PCBM film to produce ordered nanowires with diameter and periodicity corresponding to the pores on the surface of the stamp. (g) A finished sample: a silver nanovoid array in intimate contact with a layer of PCBM.

spectroscopy sample due to thin-film interference effects and ITO's non-negligible absorption. The samples on ITO-covered glass and quartz glass otherwise undergo identical production. The structuring of the organic layer via imprinting is shown schematically in Fig. 1(f). Samples $A$ and $B$ [Figs. 1(b) and 1(c)], imprinted with stamps $A$ and $B$, respectively, exhibit nanowire size, ordering, and periodicity (60 and $100 \mathrm{~nm})$ consistent with the hole array of the stamps. A third sample is produced under identical spin-coating conditions. Instead of an imprinting step, the sample undergoes an annealing treatment at the same temperature and for the same duration as the imprinting process, acting as a planar sample for comparison with samples $A$ and $B$. Finally, silver is thermally evaporated on top of the structured [Fig. 1(g)] and planar PCBM layers. Despite depositing more than $200 \mathrm{~nm}$ of silver, a discernible hexagonally packed array of mounds across the top surface of the structured samples is observable in SEM pictures (not shown). This is consistent with surface modulation arising from deposition on a structured substrate. When samples are cleaved in preparation for cross-section SEM imaging, the nanovoid array can be viewed directly in positions where the silver film has separated from the PCBM film during cleavage [Fig. 1(d)].

Samples $A$ and $B$ display striking differences in the geometry of the PCBM nanowire array. In addition to size and periodicity, the nanowires in samples $A$ and $B$ also vary in shape. Whereas the typical nanowire in sample $A$ [Fig. 1(b)] approximates a cylinder with aspect ratio 1, the typical nanowire in sample $B$ [Fig. 1(c)] more closely approximates a cone with slanted orientation in respect to the substrate. This shape is attributed to a slight deformation of the imprinted pattern in PCBM, which is in a molten and viscous state at $120{ }^{\circ} \mathrm{C},{ }^{18}$ upon demolding. It is important to note the fine size of the features. Sample $A$ exhibits a highly ordered array of nanovoids of diameter $40 \mathrm{~nm}$, height $40 \mathrm{~nm}$, and 
periodicity $60 \mathrm{~nm}$. An array of nanovoids of such small size with a high degree of order is beyond the capabilities of many other techniques. Highly ordered arrays of metallic nanovoids fabricated using the polymer sphere templating technique, for example, typically feature nanovoids of diameter much greater than $100 \mathrm{~nm}^{19}$

The reflectance of the samples is measured using a UV-Vis spectrometer (U-3501, Hitachi) fitted with an integrating sphere. The sample is positioned at an angle of $10^{\circ}$ to the incident beam. In order to minimize silver oxidation, the samples are not exposed to air between the silver deposition and the measurement. Optical and ultraviolet (UV) illumination are provided by tungsten and deuterium lamps, respectively. The light is incident on the sample through the glass side, and under the assumption that the transmission is zero, the absorbance $A$ of the system can be obtained from the reflectance $R$ using $A=1-R$.

\section{RESULTS AND DISCUSSION}

\section{A. Reflectivity measurements}

The experimentally obtained absorption spectra are shown in Fig. 2. Each spectrum exhibits maximum absorption in the range $300-330 \mathrm{~nm}$, which is primarily attributed to absorption in silver. This spectral position corresponds to silver's bulk plasmon resonance. ${ }^{20,21}$ In the context of this system, the strong absorption in this wavelength range can be understood to arise from a sharp decrease in the real part of silver's refractive index and hence an increase in transmission at the PCBM-Ag interface. At wavelengths beyond this peak, where (planar) silver typically exhibits near-perfect reflection, absorption features that are attributed to PCBM are observed. A clear enhancement in total absorption over the planar sample is observed for both of the nanostructured samples. For the 300-800-nm wavelength range, the absorption is increased in samples $A$ and $B$ by $15 \%$ and $80 \%$, respectively. It is important to note that the total volume of PCBM in the nanostructured sample does not exceed that of the planar sample. In fact, it is slightly less, as a small amount of PCBM invariably adheres to the stamp during demolding, despite anti-adhesion

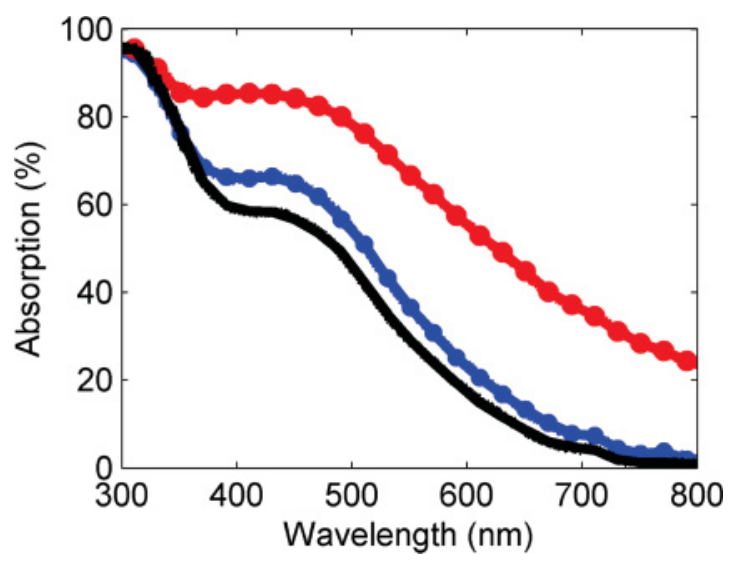

FIG. 2. (Color online) Absorption spectra of planar (black line), nanostructured with feature periodicity $60 \mathrm{~nm}$ (small blue circles) and nanostructured with feature periodicity $100 \mathrm{~nm}$ (large red circles) samples as obtained from reflection spectroscopy measurements. measures being taken. Nevertheless, broad enhancements in total absorption (the contribution of which is attributed to PCBM will be discussed later in this paper) are observed for the nanostructured samples. In sample $A$, with a $60-\mathrm{nm}$ feature periodicity, the enhancement is concentrated within the absorption range of PCBM, with a peak enhancement at $430 \mathrm{~nm}$. For sample $B$, which has a $100-\mathrm{nm}$ feature periodicity, a significantly higher level of light trapping is observed. The absorption enhancement observed for sample $B$ extends well beyond the absorption edge of PCBM (around $750 \mathrm{~nm}$ ). We conclude therefore that both the absorption beyond the absorption edge and a significant amount of absorption below it must occur within the silver layer. This significant coupling to the silver layer as a result of the interface nanostructure could be facilitated by excitation of surface modes or an enhanced transmission through the interface. In order to obtain an insight into the absorption enhancement mechanisms governing the behavior of a PCBM-silver nanovoid interface, three-dimensional full-wave simulations have been carried out.

\section{B. Finite element simulations}

The finite element method (FEM), invoked with commercially available software (COMSOL), is used to numerically solve Maxwell's equations and thus compare the optical field distributions in systems featuring a planar and a nanovoid array interface between silver and PCBM. Whereas a completely enclosed void can be accurately treated using Mie theory, numerical techniques are generally required to investigate plasmonic behavior in metallic nanovoids with more arbitrary geometries. Techniques that have been previously invoked to address arbitrary nanovoid geometries include the boundary element method $^{22}$ and the finite-difference time-domain method. ${ }^{23}$ The FEM is appealing because it is a full-wave technique and supports arbitrary geometries. In addition, as it is a method that operates in the frequency domain, experimentally determined material dispersion can be conveniently incorporated without the need to model the optical constants, as is required when using the finite-difference time-domain method. The FEM has been widely used to investigate plasmonic structures such as SPP waveguides, metallic nanoshells, and nanorod arrays. ${ }^{24-26}$

The system consists of three layers: glass, PCBM, and silver. Values for the complex refractive indices of PCBM and silver are obtained from the literature, ${ }^{27,28}$ and the glass is assigned a refractive index of 1.4. Surface roughness at the two interfaces is neglected, and the materials are assumed to be optically isotropic.

The PCBM-silver interface is defined in the $x-y$ plane, with plane-wave illumination from normal incidence (along the $z$ direction), as shown in Fig. 3(a). Circularly polarized incident light is used. As the magnitude of the electric field vector in circularly polarized light is preserved as it rotates in the plane of polarization, this is a close approximation of the unpolarized illumination of the lamp (and sunlight) - the time-averaged polarization is zero in both cases. Periodic boundary conditions are applied to all four side boundaries. The system is terminated at the illumination side with a perfectly matched layer, ${ }^{29}$ designed to absorb all reflected light and thus minimize artificial reflections. The planar interface is modeled with a PCBM layer thickness of $75 \mathrm{~nm}$. The 
(a)
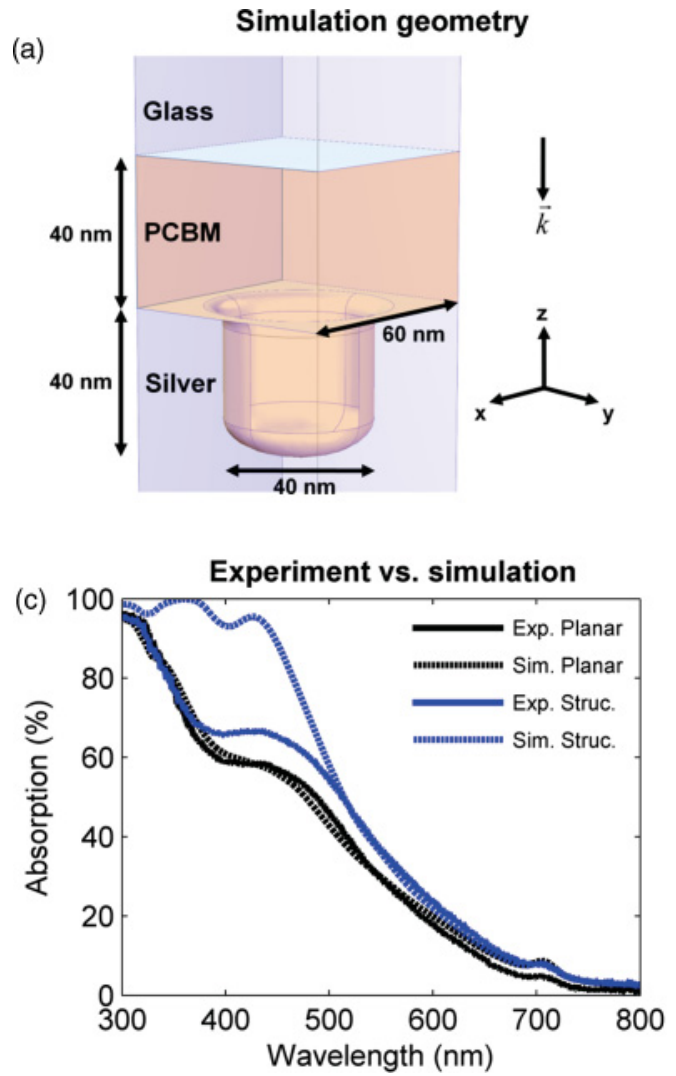
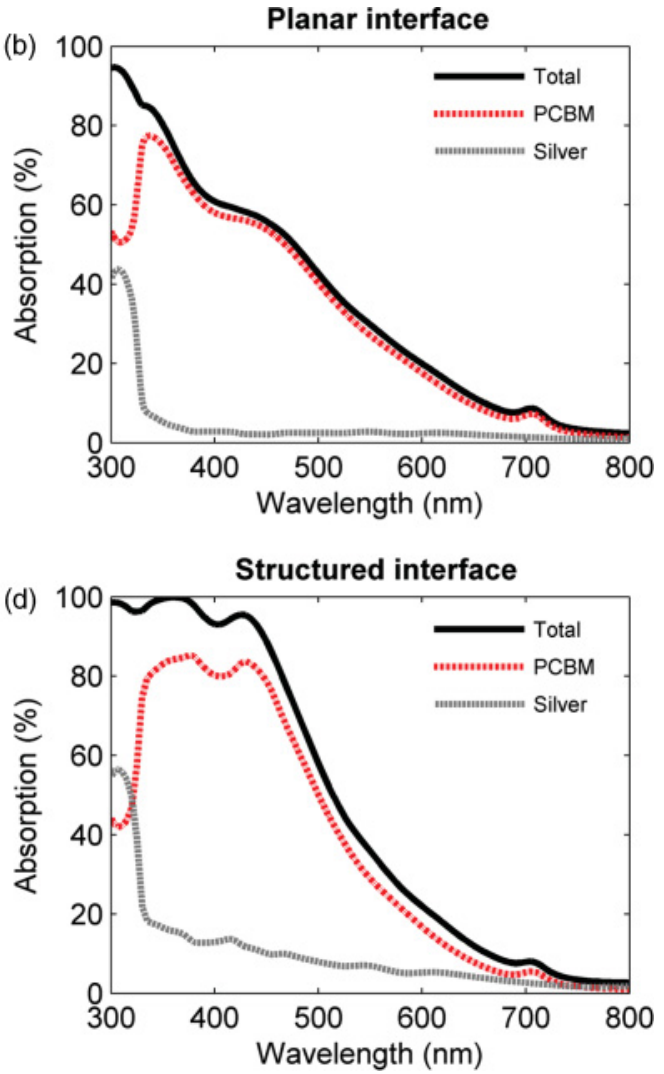

FIG. 3. (Color online) (a) The simulation geometry of a single void. The plane wave is incident from the glass side. (b) The agreement between the experimental and simulated total absorption for the planar and structured system, where the structured system refers to sample $A$ (60-nm feature periodicity). (c)-(d) The simulated contributions from PCBM and silver to the total absorption in the systems featuring a planar (c) and structured (d) interface. Important features that arise due to the structured interface include the emergence of two new peaks in PCBM absorption at 375 and $430 \mathrm{~nm}$.

structured interface is modeled with size parameters chosen to resemble sample $A$ [Fig. 1(b)], whose symmetric structure is conducive to accurate simulation (corresponding simulations of the asymmetric nanovoids, sample $B$, are included as supplementary material ${ }^{30}$ ). Each PCBM nanowire is modeled as a cylinder with a height and diameter of $40 \mathrm{~nm}$ [Fig. 3(a)]. The tip is rounded using a 10-nm-high oblate spheroid cap, and the join to the bulk PCBM layer is softened with a curved beveled edge of radius $5 \mathrm{~nm}$. The thickness of the underlying, planar PCBM layer is set to $40 \mathrm{~nm}$. All of these values are based on an analysis of SEM pictures [such as Fig. 1(b)] using an image analysis software (ImageJ). Square and hexagonal lattices are simulated by appropriate placement of one or multiple nanovoids (one and four, respectively) in appropriate periodic cells. Unless otherwise indicated, a hexagonal lattice is used, consistent with the experimental samples.

The time-averaged energy absorption rate in each material is calculated using the expression: $\frac{1}{2} \int \omega \varepsilon_{2}|E|^{2} d V$, where the integral is evaluated over the material volume $V, \varepsilon_{2}$ the imaginary part of the permittivity, and $\omega$ and $E$ the angular frequency and electric field strength of the electromagnetic field, respectively. As a check, the reflectance of the composite system is calculated independently as a flux integral over the boundary separating the system and the perfectly matched layer. The sum of the independently calculated system absorption and reflection is well within $0.1 \%$ of unity for all simulated wavelengths for planar as well as structured interface geometries.

The routine was rigorously tested to confirm the validity of the calculations and to quantify the strength of the conclusions that can be drawn from the results. Absorption and reflection spectra for arbitrary planar multilayer systems agree exactly with those calculated using transfer-matrix formalism. ${ }^{31}$ Excellent agreement with experimentally determined total absorption of the planar system is observed [see Fig. 3(b), black curves]. The slight discrepancy between the experimental and simulated spectra can be attributed to a number of factors including the small offset in incidence angle between the experimental and modeled system and the assumption of smooth interfacial surfaces. The relative contributions of the PCBM and silver layers to the total absorption are plotted in Fig. 3(c). The typical mirror-like nature of planar silver is observed, with excellent reflection properties in the optical and near infra-red wavelength region. Accordingly, absorption in the PCBM at these wavelengths accounts for almost all of the total absorption. At lower wavelengths the mirror-like property is no longer as observable. As mentioned earlier, the transmittance at the PCBM-Ag interface becomes large at wavelengths close to the bulk plasmon frequency, contributing to a sharp peak in absorption in the silver layer. This causes a corresponding dip in the PCBM absorption at these wavelengths. Above $330 \mathrm{~nm}$, the PCBM absorption spectrum 
displays the typical shape observed in the complex part of its refractive index, ${ }^{27}$ including the main peak at around 340 $\mathrm{nm}$, a shoulder at around $450 \mathrm{~nm}$, and a small peak at around $700 \mathrm{~nm}$. Given the very good agreement between simulation and experiment for the planar case, it is reasonable to infer that the PCBM and silver absorption contributions to the experimentally determined total absorption (Fig. 2) are similar to those shown in Fig. 3(c).

As shown in Fig. 3(b), we also observe good agreement between the simulated and experimentally determined absorption spectra for the structured interface (blue/gray lines), although the agreement is not as close as is it is for the planar case. This discrepancy will be discussed in more detail later in this subsection. We note, however, that the enhancement in absorption over the planar case is clearly reproduced. Importantly, the wavelength of the maximum enhancement in absorption, $430 \mathrm{~nm}$, coincides exactly with the position of a peak in the simulated spectrum. The relative contributions of the PCBM and silver layers to the total absorption for the structured case are plotted in Fig. 3(d). The general behavior is similar to that of the planar system, although there are some important differences. Given that the structured interface is capable of hosting localized plasmonic modes, the observed overall increase in the absorption of the system is expected. The spatial distribution of this additional absorption is highly dependent on the nature of the excitation and the refractive indices of the two materials. This has been calculated to be strongly concentrated in the organic layer for SPP modes on silver-organic semiconductor interfaces. ${ }^{4}$ In the system simulated here, the absorption in silver has increased significantly across the spectrum, in particular in the UV region. Importantly, however, the PCBM absorption has also significantly increased. Two additional peaks are evident, one at $375 \mathrm{~nm}$, which is easily resolved from the $340 \mathrm{~nm}$ PCBM absorption peak; and a second at $430 \mathrm{~nm}$, which, as mentioned above, coincides with the wavelength of maximum enhancement in the experimental absorption spectrum.

The variation of the $y$ component of the electric field (Fig. 4) over time reveals the nature of the peaks observed

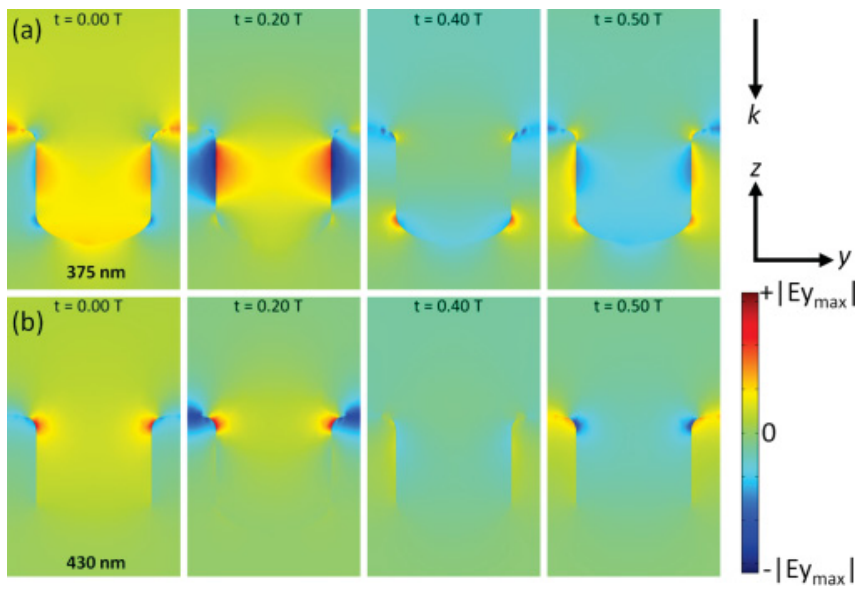

FIG. 4. (Color online) Simulated $y$ component of the electric field at (a) 375 and (b) $430 \mathrm{~nm}$ for times $0,0.2,0.4$, and $0.5 \mathrm{~T}$, respectively, where $\mathrm{T}$ is the period of the oscillation of the incident wave. The linear color scale indicates the strength of the field relative to the maximum and minimum values observed at each wavelength. in Fig. 3. These profiles, displaying the behavior at four points in time across half of the periodic cycle $T$ (see supplementary material for movies that show the complete time dependence), ${ }^{32}$ are taken across a plane $x=$ constant, which bisects a row of nanovoids separated by the lattice period. Similar plots are obtained regardless of whether the voids are ordered in square or hexagonal lattices. Absorption at wavelengths corresponding to peaks in the PCBM absorption spectrum is highly localized at the interface, characteristic of surface-bound plasmonic excitation. The short-wavelength peak [Fig. 4(a)] corresponds to enhanced electric field strength along the inner walls of the voids. The localization at this wavelength is evidently achieved via the excitation of a standing wave along the rim of the nanovoid, which has been proposed previously, but for much larger voids. ${ }^{19,33}$ Antinodes are observed on the nanovoid sides and shoulders, separated by nodes. The amplitude of the surface mode decreases along the length of the void, which we attribute to absorption in the semiconductor and metal (plotted explicitly in Fig. 5). We note that the void diameter is much smaller than the wavelength of incident light; penetration is only possible due to the excitation of this surface mode. At the longer wavelength [Fig. 4(b)], light does not significantly penetrate into the voids; rather, an enhanced electric field is observed along the surface between adjacent voids.

Time-averaged absorption profiles for polarization along the (10) crystal direction are extracted to illustrate the relationship between the mode absorption and the orientation of the electric field vector of the incident light (Fig. 5). The profiles are plotted in three different planes for the structured case: the $y-z$ plane $(x=0), x-z$ plane $(y=0)$, and the $x-y$ plane $(z=-2 \mathrm{~nm})$, respectively. The profile in the $x-y$ plane is taken within the silver layer, as opposed to directly at the interface.

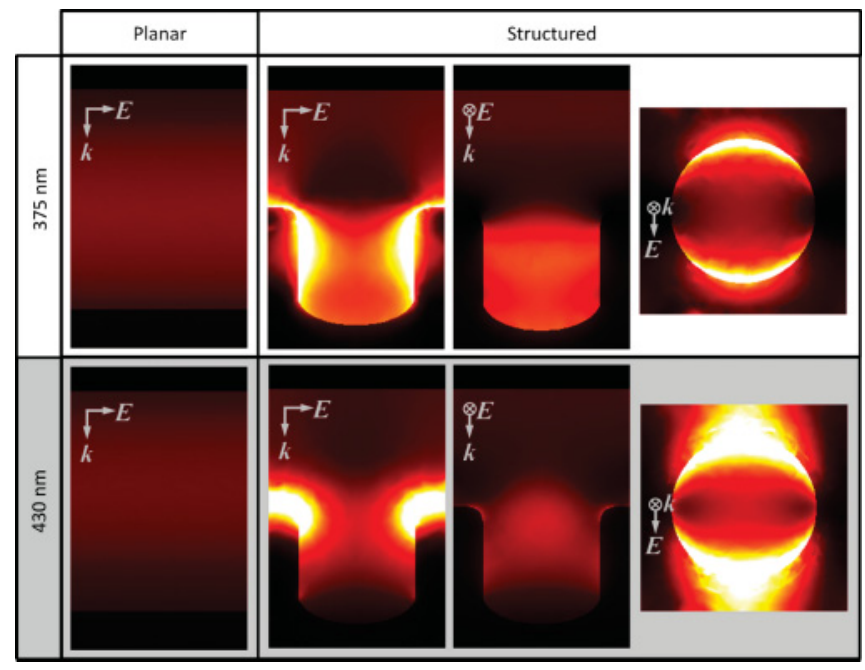

FIG. 5. (Color online) Absorption rate profiles (linear color scale) for wavelengths 375 and $430 \mathrm{~nm}$. Black indicates areas of zero absorption, and white indicates areas of maximum absorption. For each wavelength, the planar and structured interfaces are compared. For the structured interface, the absorption rate profile is shown in three different planes, as indicated relative to the electric field vector $E$ and propagation vector $k$. For each wavelength displayed, significantly larger absorption, which is strongly spatially localized in nature, is observed in the structured system. 


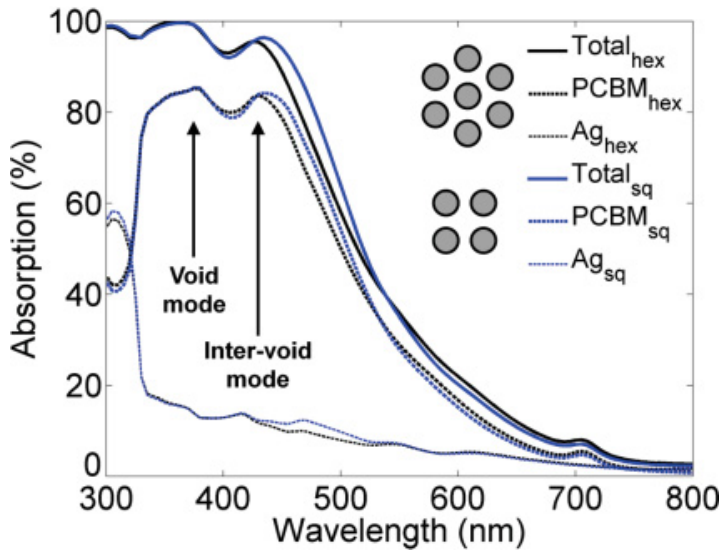

FIG. 6. (Color online) The effect of the void lattice type (hexagonal or square) on light dissipation.

The antinodes of the standing wave responsible (Fig. 4) for the short wavelength peak $(375 \mathrm{~nm})$ can be observed but not clearly resolved, and the amplitude of the wave (and hence the mode absorption) is seen to decrease along the length of the void towards the base. The relatively insignificant penetration of the long-wavelength mode $(430 \mathrm{~nm})$ compared to the short-wavelength mode can clearly be seen. Localized absorption is entirely absent in the system with the planar interface. Excitation of both void and inter-void modes is observed most prominently along the direction of the electric field. The absorption profile for circularly polarized incident light (not shown) is accordingly symmetric about the $z$ axis.

Similar behavior is observed regardless of whether the voids are ordered in square or hexagonal arrays (Fig. 6). The short-wavelength peak has been shown to be due to strong localized absorption within the void [Fig. 4(a)], and therefore its spectral position is insensitive to the void ordering. We see that the magnitude of this peak is identical for square and hexagonal lattices despite the increased $(15 \%)$ void-packing density of the hexagonal lattice. This is because the total absorption at this wavelength is already saturated for the square lattice; increasing the density of voids cannot increase the total absorption of the system beyond $100 \%$. In the hexagonal case, the light trapped in this mode per void decreases compared to the square case, with the relative absorption in the semiconductor and silver remaining unchanged. The long-wavelength peak corresponds to localization between the voids [Fig. 4(b)], and we observe a weak dependency on lattice order, or in other words, on the position of the nearest neighboring voids. The width of this peak is slightly broadened for the square array, a result of the reduced rotational symmetry of the square array compared to the hexagonal array. However, it must be stressed that the overall difference between the two configurations is small: the total absorption for the hexagonal lattice, spectrally integrated over the investigated wavelength region, is higher than that of the square lattice by less than $1 \%$.

Simulations (for the square lattice, for example) where the polarization vector is held constant over time indicate an identical response regardless of whether the electric field is aligned along the $(1,0)$ (void spacing of the lattice constant $a$ ) or $(1,1)$ (void spacing of $\sqrt{2} a$ ) crystal directions. Polarization independence (at normal incidence) has also been observed in similar systems, such as square arrays of holes ${ }^{34}$ in metal films and hexagonal arrays of silver nanoparticles.$^{35}$ Combined with the result of Fig. 6-the response is similar for square and hexagonal lattices-we see that the placement of neighboring nanovoids is of minor importance for this configuration (60-nm lattice constant and 40-nm void diameter): The long-wavelength mode does not significantly extend beyond the distance to the next void to the extent that the lattice order is of major importance, and the short-wavelength mode exists entirely within the voids themselves. The response can effectively be considered as the sum of the responses of noninteracting nanovoids rather than a collective response of interacting nanovoids.

We will now discuss the agreement between experimentally determined and simulated absorption spectra for the structured interface system and attempt to account for the discrepancy [Fig. 3(b), blue/gray lines]. Deviations between experimental and simulated structures are proposed as the primary cause of this discrepancy. As stated earlier, the most significant enhancement in absorption occurs at around $430 \mathrm{~nm}$ for both the simulated and experimental spectra. However, the absorption peak at this wavelength is decidedly sharper in the simulated spectrum - a consequence of the perfect uniformity in the shape of the simulated voids and the irregularity of the voids across the experimental sample. The wavelengths at which plasmonic modes can be efficiently excited in nanovoid systems are highly dependent on the void geometry. ${ }^{10}$ Therefore, a distribution in void size and shape around a mean value, and a corresponding distribution of wavelengths at which the plasmonic modes are efficiently excited, would have the observed effect of broadening the absorption peak. This effect is expected to be exacerbated for sample $B,{ }^{30}$ where the asymmetry and variation in shape is increased [Fig. 1(c)].This significantly increases the difficulty of simulation. The higher absorption of sample $B$ is an indication that increased variation of plasmonic hosting features is advantageous for obtaining broadband light trapping, which is the principle behind tandem plasmonic devices. ${ }^{4}$ For rigorous reports on the effect of void shape and size on plasmonic excitation, we refer the reader to the literature. ${ }^{10,22}$ A second reason for the discrepancy is that, due to inherent surface modulation in both the PCBM film and the stamp, a finite area of PCBM will have insufficient contact to the stamp during imprinting. The sample area is therefore made up of positions displaying a perfect imprint and positions where the imprint is either less prominent or absent. The absorption of such a system could be given as an average of the absorption of the structured and planar systems, appropriately weighted by the fractional area of each. This correction would have the effect of making the absorption spectrum for the structured case more similar to that of the planar case, as is observed in the measured data. Despite these difficulties, the observed overall agreement between experiment and simulation is quite strong. Even better agreement is expected if the order and regularity of the experimental structures is further improved.

\section{CONCLUSION}

In conclusion, significant light trapping has been demonstrated in a system featuring an organic semiconductor adjacent to an ordered metallic nanovoid array. In order 
to realize these structures, an imprinting technique was employed. The applicability of this technique extends to all materials that are soft enough to be imprinted, and the flexibility in pore diameter, depth, and periodicity allows a degree of application-specific tuning. The fabricated nanostructured interfaces feature hexagonally packed arrays of silver nanovoids, upon which localized and propagating surface plasmons can be excited. Using two different nanovoid geometries, featuring nominal void periodicity of 60 and $100 \mathrm{~nm}$, respectively, we observed total absorption enhancements of $15 \%$ and $80 \%$ across the wavelength region $300-800 \mathrm{~nm}$. Three-dimensional, full-wave simulations using the finite element method were invoked to contrast and gain insight into the mechanisms governing absorption in nanovoid- and planar-interface geometries. Very good agreement is observed between experimentally determined and simulated absorption spectra. Simulation results indicate that a significant fraction of the total additional absorption occurs in the organic semiconductor. Energy absorption rate profiles obtained from the simulations, which reveal strongly localized absorption at the metal-semiconductor interface, provide strong evidence that the origin of the observed light trapping is indeed due to the excitation of surface-bound plasmonic modes. These results suggest that by substituting planar metallic electrodes in organic solar cells with the nanostructured electrodes presented here, the exciton generation rate can be strongly increased.

\section{ACKNOWLEDGMENTS}

We gratefully acknowledge the International Doctorate Scholarship Program, a part of the Elite Network of Bavaria, the German Research Foundation, and the Cluster of Excellence Nanosystems Initiative Munich. We would like to thank W. Töllner and S. Heiderich in the Professor Nielsch Group (University of Hamburg, Germany) for their support with AAO production. We thank J. Kotthaus and J. Feldmann for providing access to various instruments, and $\mathrm{H}$. Hoppe for providing the optical constants for PCBM.
*Corresponding author: Department of Physics, University of Konstanz, Fach M 680, D-78457 Constance, Germany; Lukas. schmidt-mende@uni-konstanz.de

${ }^{1}$ T. W. Ebbesen, H. J. Lezec, H. F. Ghaemi, T. Thio, and P. A. Wolff, Nature 391, 667 (1998).

${ }^{2}$ S. M. Nie and S. R. Emery, Science 275, 1102 (1997).

${ }^{3}$ J. Weickert, R. B. Dunbar, H. C. Hesse, W. Wiedemann, and L. Schmidt-Mende, Adv. Mater. 23, 1810 (2011).

${ }^{4}$ H. A. Atwater and A. Polman, Nat. Mater. 9, 205 (2010).

${ }^{5}$ D. Derkacs, S. H. Lim, P. Matheu, W. Mar, and E. T. Yu, Appl. Phys. Lett. 89, 093103 (2006).

${ }^{6}$ K. Nakayama, K. Tanabe, and H. A. Atwater, Appl. Phys. Lett. 93, 121904 (2008).

${ }^{7}$ J. H. Lee, J. H. Park, J. S. Kim, D. Y. Lee, and K. Cho, Org. Electron. 10, 416 (2009).

${ }^{8}$ J. L. Wu, F. C. Chen, Y. S. Hsiao, F. C. Chien, P. L. Chen, C. H. Kuo, M. H. Huang, and C. S. Hsu, Acs Nano 5, 959 (2011).

${ }^{9}$ S. A. Maier, Plasmonics: Fundamentals and Applications (Springer, New York, 2007), p. 46.

${ }^{10}$ T. A. Kelf, Y. Sugawara, R. M. Cole, J. J. Baumberg, M. E. Abdelsalam, S. Cintra, S. Mahajan, A. E. Russell, and P. N. Bartlett, Phys. Rev. B 74, 245415 (2006).

${ }^{11}$ A. P. Li, F. Muller, A. Birner, K. Nielsch, and U. Gosele, J. Appl. Phys. 84, 6023 (1998).

${ }^{12}$ W. Wiedemann, L. Sims, A. Abdellah, A. Exner, R. Meier, K. P. Musselman, J. L. MacManus-Driscoll, P. Müller-Buschbaum, G. Scarpa, P. Lugli, and L. Schmidt-Mende, Appl. Phys. Lett. 96, 263109 (2010).

${ }^{13}$ H. C. Hesse, D. Lembke, L. Dossel, X. Feng, K. Mullen, and L. Schmidt-Mende, Nanotechnology 22, 055303 (2011).

${ }^{14}$ M. Aryal, F. Buyukserin, K. Mielczarek, X. M. Zhao, J. M. Gao, A. Zakhidov, and W. C. Hu, J. Vac. Sci. Technol. B 26, 2562 (2008).

${ }^{15}$ X. M. He, F. Gao, G. L. Tu, D. Hasko, S. Huttner, U. Steiner, N. C. Greenham, R. H. Friend, and W. T. S. Huck, Nano Lett. 10, 1302 (2010).

${ }^{16}$ W. Lee, R. Ji, U. Gosele, and K. Nielsch, Nat. Mater. 5, 741 (2006).
${ }^{17}$ D. C. Duffy, J. C. McDonald, O. J. A. Schueller, and G. M. Whitesides, Anal. Chem. 70, 4974 (1998).

${ }^{18}$ J. Zhao, A. Swinnen, G. van Assche, J. Manca, D. Vanderzande, and B. V. Mele, J. Phys. Chem. B 113, 1587 (2009).

${ }^{19}$ T. A. Kelf, Y. Sugawara, J. J. Baumberg, M. Abdelsalam, and P. N. Bartlett, Phys. Rev. Lett. 95, 116802 (2005).

${ }^{20}$ A. Liebsch, Phys. Rev. Lett. 71, 145 (1993).

${ }^{21}$ M. Rocca, Surf. Sci. Rep. 22, 45 (1995).

${ }^{22}$ R. M. Cole, J. J. Baumberg, F. J. Garcia de Abajo, S. Mahajan, M. Abdelsalam, and P. N. Bartlett, Nano Lett. 7, 2094 (2007).

${ }^{23}$ V. E. Ferry, L. A. Sweatlock, D. Pacifici, and H. A. Atwater, Nano Lett. 8, 4391 (2008).

${ }^{24}$ T. Holmgaard and S. I. Bozhevolnyi, Phys. Rev. B 75, 245405 (2007).

${ }^{25}$ C. G. Khoury, S. J. Norton, and T. Vo-Dinh, Nanotechnology 21, 315203 (2010).

${ }^{26}$ A. Wei, D. P. Lyvers, J. M. Moon, A. V. Kildishev, and V. M. Shalaev, Acs Nano 2, 2569 (2008).

${ }^{27}$ H. Hoppe, N. S. Sariciftci, and D. Meissner, Mol. Cryst. Liq. Cryst. 385, 233 (2002).

${ }^{28}$ P. B. Johnson and R. W. Christy, Phys. Rev. B 6, 4370 (1972).

${ }^{29}$ J. P. Berenger, J. Comput. Phys. 114, 185 (1994).

${ }^{30}$ See Supplemental Material at http://link.aps.org/supplemental/ 10.1103/PhysRevB.85.035301 for simulations of the asymmetric nanovoids.

${ }^{31}$ Transfer-matrix calculations are performed with Freesnell, a thin-film optical simulator [http://people.csail.mit.edu/jaffer/ FreeSnell/]

${ }^{32}$ See Supplemental Material at http://link.aps.org/supplemental/ 10.1103/PhysRevB.85.035301 for movies showing the full timedependence of these excitations.

${ }^{33}$ N. M. B. Perney, J. J. Baumberg, M. E. Zoorob, M. D. B. Charlton, S. Mahnkopf, and C. M. Netti, Opt. Express 14, 847 (2006).

${ }^{34}$ J. Braun, B. Gompf, G. Kobiela, and M. Dressel, Phys. Rev. Lett. 103, 203901 (2009).

${ }^{35}$ J. F. Zhu, M. Xue, H. J. Shen, Z. Wu, S. Kim, J. J. Ho, A. Hassani-Afshar, B. Q. Zeng, and K. L. Wang, Appl. Phys. Lett. 98, 151110 (2011). 